\title{
Acute Visual Loss as a First Sign of Hyperhomocysteinaemia
}

\author{
Aggeliki Kolea, ${ }^{1}$ Aggelos Baltatzidis, ${ }^{2}$ Vasileios Margaritis, ${ }^{1}$ Belal Almoghrabi, ${ }^{1}$ \\ Irini Kaldi, ${ }^{1}$ Alexandros Polychronakos ${ }^{1}$ \\ ${ }^{1}$ Department of Ophthalmology, Agios Dimitrios General Hospital, and ${ }^{2}$ Department of \\ Radiology, AHEPA Hospital, Aristotle University, Thessaloniki, Greece
}

\begin{abstract}
A 38-year-old man presented with an acute right homonymous visual field defect due to occipital lobe infarct caused by hyperhomocysteinaemia. Visual field testing, magnetic resonance imaging, laboratory studies, and genetic analysis were carried out. On magnetic resonance imaging, a left occipital lesion with bright signal on the diffusion-weighted and fluid-attenuated inversion recovery images suggested a diagnosis of an acute infarct. Blood tests revealed raised homocysteine of $52.08 \mu \mathrm{mol} / \mathrm{L}$ (reference range, $<15 \mu \mathrm{mol} / \mathrm{L}$ ) and genetic analysis showed the patient to be homozygote to 5, 10-methylenetetrahydrofolate reductase deficiency. Hyperhomocysteinaemia is a rare causes of acute visual loss due to cerebral ischaemia and should always be suspected and investigated with the appropriate tests to diagnose the condition and limit further vision deterioration.
\end{abstract}

Key words: Hyperhomocysteinemia, Magnetic resonance imaging, Visual fields

Asian J Ophthalmol. 2011;12:172-4.

\section{Introduction}

Homocystinuria is a disorder of methionine metabolism, leading to abnormal accumulation of homocysteine and its metabolites such as homocystine and homocysteine-cysteine complex in blood and urine. Normally, these metabolites are not found in appreciable quantities in blood or urine. Hyperhomocysteinaemia, a separate but related entity, is defined as elevation of homocysteine level in blood. The reported incidence of homocystinuria varies between 1 in 50,000 and 1 in 200,000 . The prevalence of hyperhomocysteinaemia in the general population is not known. Studies investigating the prevalence of homozygosity for the thermolabile variant mutation in 5, 10-methylenetetrahydrofolate reductase (MTHFR) have found a prevalence of approximately $15 \%$ in European, Middle Eastern, and Japanese populations compared with a prevalence of $\leq 1.4 \%$ in African Americans. The prevalence of heterozygosity is $30 \%$ to $40 \%$.

This report is of a 38-year-old man who, with no other risk factors, presented with an acute right homonymous visual field defect as the first sign of hyperhomocysteinaemia.

\section{Case Report}

A 38-year-old man presented to the eye casualty because of sudden-onset visual field loss of his right eye, accompanied

Correspondence: Dr Vasileios Margaritis, Flat 12, 3 Queensborough Terrace, London, W2 3TA, UK.

Tel: 447747811154 ;

E-mail: vmargaritis@yahoo.gr or vmargaritis@gmail.com by a mild non-localized headache. He had no prior medical or significant family history. He was not taking any medications and did not smoke. His visual acuity was 20/20 in the right eye and $20 / 20$ in the left eye. Colour vision, anterior and posterior segment examination, intraocular pressure, and pupillary responses were normal. Neurological examination was normal except for the visual field, which revealed a right homonymous hemianopia that was densest in the superior quadrant (Figure 1). The differential

Figure 1. Visual fields showing right homonymous hemianopia (a) at presentation; and (b) after 2 months.

(a)
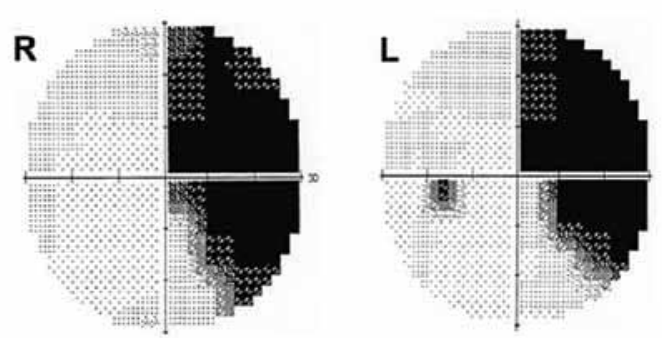

(b)
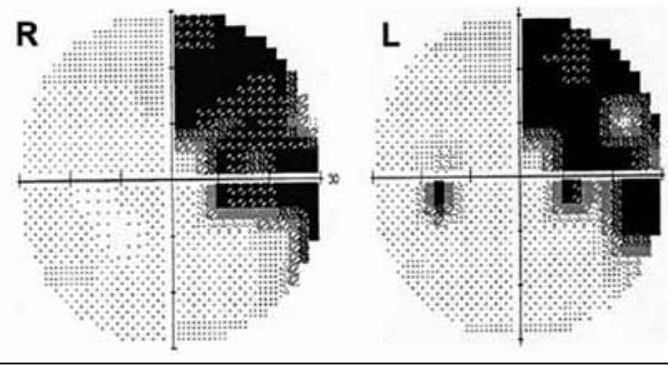
Figure 2. Magnetic resonance imaging of the brain depicting cerebral infarction. (a) 24 hours after onset of hemianopia onset, from left to right: diffusionweighted, FLAIR, and gadolinium-enhanced T1-weighted axial images demonstrating an infarct of the left occipital Iobe with high intensity signal on diffusion-weighted and fluid-attenuated inversion recovery images and low intensity signal without abnormal enhancement on T1-weighted image (arrows); and (b) after 4 months, from left to right: fluid-attenuated inversion recovery image showing persistent hyperintensity in the left occipital lobe and nonenhanced T1-weighted image demonstrating cortical laminar necrosis represented by cortical high intensity signal (arrow).

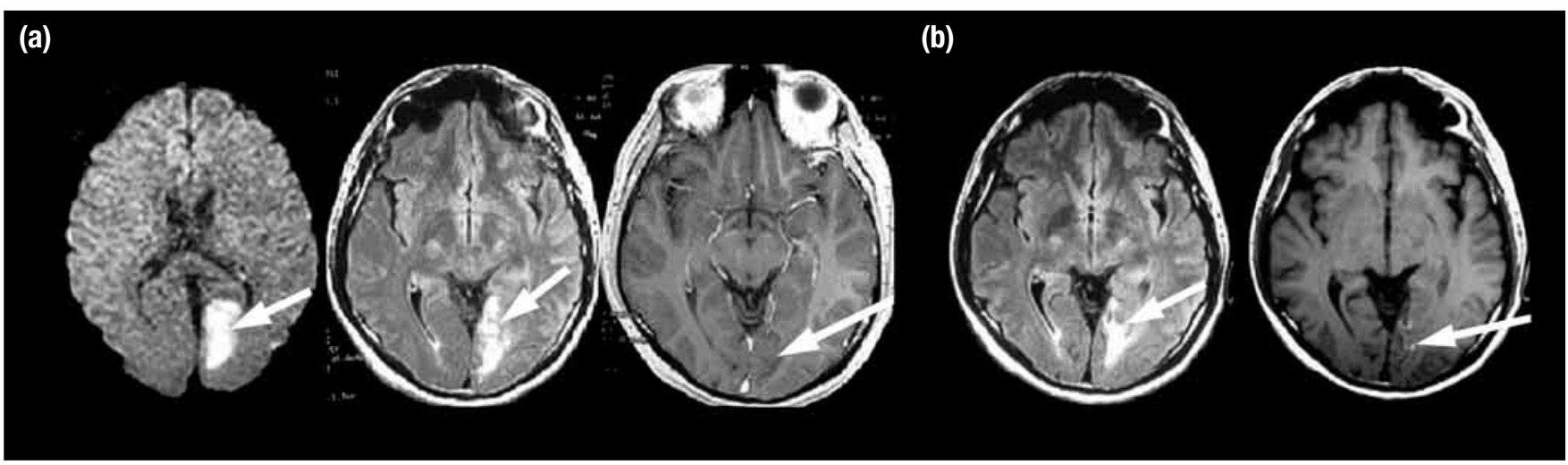

diagnosis included stroke (ischaemic or haemorrhagic), vascular malformation, space-occupying lesion, demyelinating disease, and migraine. $^{1,2}$

Urgent magnetic resonance imaging (MRI) showed an occipital infarct on diffusion-weighted and fluid-attenuated inversion recovery images (Figure 2). The patient was admitted to the Department of Ophthalmology, Agios Dimitrios General Hospital, Thessaloniki, Greece, and treated with antiplatelet drugs according to the international standards. ${ }^{3,4}$ Carotid artery triplex ultrasound and echocardiogram (transoesophageal and transthoracic) did not show any abnormalities. Laboratory studies, including evaluation of coagulation (prothrombin time, activated partial thromboplastin time, thrombin time, and bleeding time) and immunological status (antinuclear antibodies and antineutrophil cytoplasmic antibodies), revealed raised levels of homocysteine in the blood of $52.08 \mu \mathrm{mol} / \mathrm{L}$ (reference range, $<15 \mu \mathrm{mol} / \mathrm{L}$ ). Genetic analysis showed the patient to be homozygote to MTHFR deficiency, a known cause for raised homocysteine blood levels. The patient was treated with clopidogrel bisulphate $75 \mathrm{mg}$ once daily, B6 and B12 vitamin supplements, and folic acid, and discharged.

At the 2-month follow-up examination, the patient felt his vision had improved. There was improvement in the visual field in the inferior and superior quadrants (Figure 1). Blood homocysteine levels had decreased and all other laboratory tests were normal.

Four months later, a new MRI revealed left occipital lobe hyperintensity of a smaller size with signs of laminar cortical necrosis (Figure 2). Visual field examination was stable with no further deterioration.

\section{Discussion}

This report is of a unique patient with an acute right homonymous visual field defect being the first sign of hyperhomocysteinaemia.
Only one similar patient has been reported in the literature which, contrary to this patient, had a risk factor of atrial septal defect. ${ }^{5}$

Homocysteine levels increase in a variety of situations including poor diet (low consumption of vitamins B6, B12, and folic acid), low levels of thyroid hormone, renal disease, psoriasis, and use of certain medicines such as fibrates. Increased homocysteine level is associated with a higher risk of strokes. Several mechanisms have been suggested as the possible cause of accelerated vascular disease, including endothelial cell damage, smooth muscle cell proliferation, lipid peroxidation, upregulation of prothrombotic factors (XII and V), and downregulation of antithrombotic factors or endothelial-derived nitric oxide. .-8 $^{6}$

Hyperhomocysteinaemia is one of the rare causes of acute visual loss due to cerebral ischaemia and should always be suspected and investigated with the appropriate tests because several studies have shown that early diagnosis and institution of treatment, as well as dietary restriction, is likely to slow the progression of disease and reverse some of the features.

This report illustrates the need for further laboratory studies in young patients who present with symptoms of acute stroke in the absence of typical risk factors such as hypertension, smoking, and diabetes, and highlights the need for neuroimaging studies for every patient with visual field loss.

\section{References}

1. Zhang X, Kedar S, Lynn MJ, Newman NJ, Biousse V. Homonymous hemianopias: clinical-anatomic correlations in 904 cases. Neurology. 2006;66:906-10.

2. Zhang X, Kedar S, Lynn M, Newman N, Biousse V. Homonymous hemianopia in stroke. J Neuroophthalmol. 2006;26:180-3.

3. Lutsep HL. Recent clinical trials of antiplatelet therapy in secondary stroke prevention: expectations and results for application in primary case settings. Am J Med. 2009;122:21-31.

4. Goldstein LB, Adams R, Alberts MJ, et al; American Heart Association/American Stroke Association Stroke Council 
Atherosclerotic Peripheral Vascular Disease Interdisciplinary Working Group; Cardiovascular Nursing Council; Clinical Cardiology Council; Nutrition, Physical Activity, and Metabolism Council; Quality of Care and Outcomes Research Interdisciplinary Working Group; American Academy of Neurology. Primary prevention of ischemic stroke: a guideline from the American Heart Association/American Stroke Association Stroke Council: cosponsored by the Atherosclerotic Peripheral Vascular Disease Interdisciplinary Working Group; Cardiovascular Nursing Council; Clinical Cardiology Council; Nutrition, Physical Activity, and Metabolism Council; and the Quality of Care and Outcomes Research Interdisciplinary Working Group: the American Academy of Neurology affirms the value of this guideline. Stroke.
2006;37:1583-633.

5. Indolfi G, Bartolini E, Trapani S, Azzari C, Resti M. Cryptogenic stroke in a boy with atrial septal defect and hyperhomocysteinemia. J Child Neurol. 2008;23:1070-1.

6. Hassan A, Hunt BJ, O'Sullivan M, et al. Homocysteine is a risk factor for cerebral small vessel disease, acting via endothelial dysfunction. Brain. 2004;127:212-9.

7. Tan NC, Venketasubramanian N, Saw SM, Tjia HT. Hyperhomocyst(e)inemia and risk of ischemic stroke among young Asian adults. Stroke. 2002;33:1956-62.

8. Madonna P, de Stefano V, Coppola A, et al. Hyperhomocysteinemia and other inherited prothrombotic conditions in young adults with a history of ischemic stroke. Stroke. 2002;33:51-6. 